\title{
Differences in amplitude among electrode locations on amplitude-integrated electroencephalograms in preterm infants
}

\author{
Toru Kato', Yuichiro Sugiyama ${ }^{2}$, Takeshi Tsuji', Seiji Hayashi', Jun Natsume ${ }^{3}$ and Akihisa Okumura ${ }^{4}$
}

INTRODUCTION: To assess differences in amplitude among electrode locations on amplitude-integrated electroencephalograms (aEEGs) in preterm infants and change therein between preterm age and term-equivalent age (TEA), we investigated aEEGs in preterm infants at both 30-32 wk post-conceptional age (PCA) and TEA.

METHODS: The median values of upper- and lower-margin amplitudes were quantitatively calculated every 5 min (MedUMA5 and Med-LMA5, respectively), and peak, median, and bottom values were extracted at each location for the trans-frontal, trans-central, trans-occipital, fronto-central, and centro-occipital electrodes.

RESULTS: In 38 clinically stable preterm infants studied, most measurement items showed significant differences among the electrode locations at both preterm age and TEA. At 30-32 wk PCA, the bottom of Med-LMA5 was significantly higher for the trans-frontal electrodes than for the trans-central electrodes. In contrast, all measurements for Med-LMA5 were significantly lower for the trans-frontal electrodes than for the trans-central electrodes.

DISCUSSION: Amplitudes on aEEGs were significantly different among the electrode locations in preterm infants, and locational differences in amplitude changed between preterm age and TEA. It is necessary to understand the differences in amplitudes among the electrode locations on aEEGs in infants to appropriately evaluate them.

$T$ he amplitude-integrated electroencephalogram (aEEG) is a simple and convenient tool for continuous brain-function monitoring that gives a compressed view of the variation in amplitude of the electroencephalogram (EEG), typically using one or two channels (1). Many studies have demonstrated the prognostic value of aEEG in term infants with hypoxic ischemic encephalopathy in association with depression of amplitude (2-4). Other studies have demonstrated a correlation between depressed aEEG activity and intraventricular hemorrhage in preterm infants (5-7). In many of these studies, single-channel records from the P3 to P4 electrodes were selected on aEEG. Recently, single-channel records from the
C3 to C4 electrodes for the detection of neonatal seizures were reported $(8,9)$. Although standard electrode placement is necessary for the precise assessment on aEEG, sometimes, a nonstandard electrode placement for the trans-frontal electrode is selected because of less hair; this may be recommended by an equipment vendor. However, the validity of such nonstandard electrode placement has been rarely examined.

Quigg et al. (10) demonstrated significant differences in amplitude between the locations of electrodes on aEEG. However, the precise difference in amplitude among the electrode locations on aEEG has been rarely examined in infants. Because the amplitude, especially that of the lower margin on aEEG, is one of the most important criteria for predicting outcome in term infants with hypoxic ischemic encephalopathy (2-4), misinterpretation resulting from inappropriate electrode placement could present a serious problem. Therefore, it is important to understand the differences in amplitude among the electrode locations on aEEG in infants for precise evaluations. We hypothesized that aEEG amplitudes would differ among the locations of electrodes in preterm infants and that these locational differences in amplitude would change between preterm age and term-equivalent age (TEA) because of maturational changes in the EEG. The aim of this study was to assess differences in amplitude among trans-frontal, transcentral, trans-occipital, fronto-central, and centro-occipital electrodes on aEEG using quantitative analyses in clinically stable preterm infants at both $30-32 \mathrm{wk}$ post-conceptional age (PCA) and TEA.

\section{RESULTS}

Patient Characteristics

Subjects had a median gestational age of 30.1 (range, 27.1-32.7) wk and a median birth weight of 1,288 (range, 664-2,158) g. Twenty-six infants were males, six were small for gestational age, with birth weights below the 10th percentile, 12 were twins, nine had respiratory distress syndrome and needed treatment with surfactant, five developed chronic lung disease, and five had symptomatic patent ductus arteriosus and needed treatment with indomethacin. However, in all subjects, 
cardiovascular and respiratory conditions were stable at the time of aEEG recording.

The aEEG at 30-32 wk PCA was obtained at a median of 9 $\mathrm{d}$ of life (range, $1-40 \mathrm{~d}$ ) and a median of $31.6 \mathrm{wk}$ PCA (range, 30.0-32.9 wk). The duration of aEEG recording was a median of $65 \mathrm{~min}$ (range, $45-100 \mathrm{~min}$ ). The aEEG at TEA was performed at a median of $56 \mathrm{~d}$ of life (range, 36-89d) and a median of $38.3 \mathrm{wk}$ PCA (range, 37-40 wk). The duration of aEEG recording was a median of $60 \mathrm{~min}$ (range, $40-120 \mathrm{~min}$ ). In all $38 \mathrm{sub}$ jects, aEEG recordings at both $30-32$ wk of PCA and TEA were obtained and could be analyzed.

\section{Difference in Amplitude Among Electrode Locations at 30-32 wk PCA}

Regarding the difference in amplitude among the locations of electrodes, the $P$ values of the Kruskal-Wallis test for the peak, median, and bottom of median values of upper-margin amplitudes every $5 \mathrm{~min}$ (Med-UMA5) and those of median values of lower-margin amplitudes every $5 \mathrm{~min}$ (Med-LMA5) were $0.020,0.063,0.231,0.004,0.002$, and $<0.001$, respectively (Table 1). The peaks of Med-UMA5, and the peaks, medians, and bottoms of Med-LMA5 showed statistically significant differences among the electrode locations. Box plots of these analyses and results for multiple comparisons are shown in Figure 1. Regarding the peaks of Med-UMA5, the transfrontal electrodes showed significantly higher amplitudes than did the trans-central electrodes. With regard to the peaks and medians of Med-LMA5, the trans-occipital electrodes showed significantly lower amplitude than did the trans-frontal and fronto-central electrodes. Regarding the bottoms of MedLMA5, the trans-frontal electrodes showed significantly higher amplitudes than did the trans-central, trans-occipital, and centro-occipital electrodes. In terms of the amplitude differences between the trans-frontal and trans-central electrodes, some measurement items of Med-UMA5 and Med-LMA5 showed significantly higher values for the trans-frontal electrodes than for the trans-central electrodes.

\section{Differences in Amplitude Among Electrode Locations at TEA}

The $P$ values of the Kruskal-Wallis test for the peak, median, and bottom of Med-UMA5 and those of Med-LMA5 were $<0.001,<0.001,0.003,<0.001,<0.001$, and $<0.001$, respectively (Table 2). All measurement items showed significant differences among the electrode locations. Box plots of these analyses and results of correction for multiple comparisons are shown in Figure 2. Regarding the peaks and medians of MedUMA, the trans-occipital electrodes showed significantly lower amplitudes than did every other electrode. With regard to the bottom values of Med-UMA5, the trans-occipital electrodes showed significantly lower amplitudes than did the transfrontal, trans-central, or fronto-central electrodes. As for the peaks and medians of Med-LMA5, the trans-frontal and transoccipital electrodes showed significantly lower amplitude than did the other electrodes. In terms of the bottom values of MedLMA5, the trans-frontal and trans-occipital electrodes showed significantly lower amplitude than did the trans-central and centro-occipital electrodes. Regarding the amplitude difference between the trans-frontal and trans-central electrodes, no value for Med-UMA5 showed a significant difference, but all values for Med-LMA5 were significantly lower for the transfrontal electrodes than for the trans-central electrodes.

\section{Changes in Locational Amplitude Differences Between Preterm Age and TEA}

There were some changes in locational amplitude differences between 30-32 wk PCA and TEA. At 30-32 wk PCA, no measurement item of Med-UMA5 was lower for the trans-occipital electrodes than for the other electrodes. However, at TEA, these values were significantly lower for the trans-occipital electrodes than for the other electrodes. Furthermore, at 30-32 wk PCA, each measurement item of Med-LMA5 was relatively higher for the trans-frontal electrodes than for the other electrodes. In contrast, at TEA, these values were significantly lower for the trans-frontal electrodes than for the other electrodes, with the exception of the trans-occipital electrode.

\section{DISCUSSION}

These findings demonstrate that the amplitude on aEEG differed significantly among the electrode locations in preterm infants and that the locational differences in amplitude changed between preterm age and TEA. In this study, many measurement items showed significant differences among the electrode locations, the exception being the median and bottom values of Med-UMA5 at 30-32 wk PCA. Using quantitative analyses, Quigg et al. (10) demonstrated that increasing interelectrode distances increased amplitude on aEEG and

Table 1. Quantitative analyses of amplitude on each location of electrodes at 30-32 wk of post-conceptional age

\begin{tabular}{lcccccc}
\hline & Trans-F & Trans-C & Trans-O & F-C & C-O \\
\hline Peak of Med-UMA5 $(\mu \mathrm{V})$ & $38.4(21.9-56.2)$ & $32.4(19.3-39.3)$ & $34.5(14.1-67.6)$ & $35.6(22.7-49.6)$ & $34.2(20.9-52.8)$ & 0.020 \\
Median of Med-UMA5 $(\mu \mathrm{V})$ & $31.9(18.3-43.7)$ & $27.3(16.9-36.7)$ & $26.6(11.5-58.2)$ & $29.8(17.0-40.8)$ & $28.3(18.5-45.9)$ & 0.063 \\
Bottom of Med-UMA5 $(\mu \mathrm{V})$ & $24.8(13.6-38.9)$ & $22.9(14.5-30.9)$ & $20.4(9.7-49.5)$ & $23.1(14.3-34.6)$ & $22.3(15.7-35.4)$ & 0.231 \\
Peak of Med-LMA5 $(\mu \mathrm{V})$ & $7.2(6.0-10.0)$ & $7.1(6.0-9.0)$ & $6.6(5.0-8.0)$ & $7.4(6.0-9.0)$ & $7.0(6.0-8.0)$ & 0.004 \\
Median of Med-LMA5 $(\mu \mathrm{V})$ & $6.3(3.8-8.4)$ & $6.1(4.6-8.2)$ & $5.7(4.3-7.8)$ & $6.2(4.9-8.0)$ & $6.0(4.7-7.6)$ & 0.002 \\
Bottom of Med-LMA5 $(\mu \mathrm{V})$ & $5.8(2.8-8.0)$ & $5.2(4.0-7.4)$ & $4.8(3.5-6.9)$ & $5.4(3.7-7.6)$ & $5.2(4.0-6.9)$ & $<0.001$ \\
\hline
\end{tabular}

All values are shown as medians (range). Trans means bilateral.

C, central; F, frontal; Med-LMA5, median value of lower-margin amplitude every 5 min; Med-UMA5, median value of upper-margin amplitude every 5 min; O, occipital.

akruskal-Wallis test. 

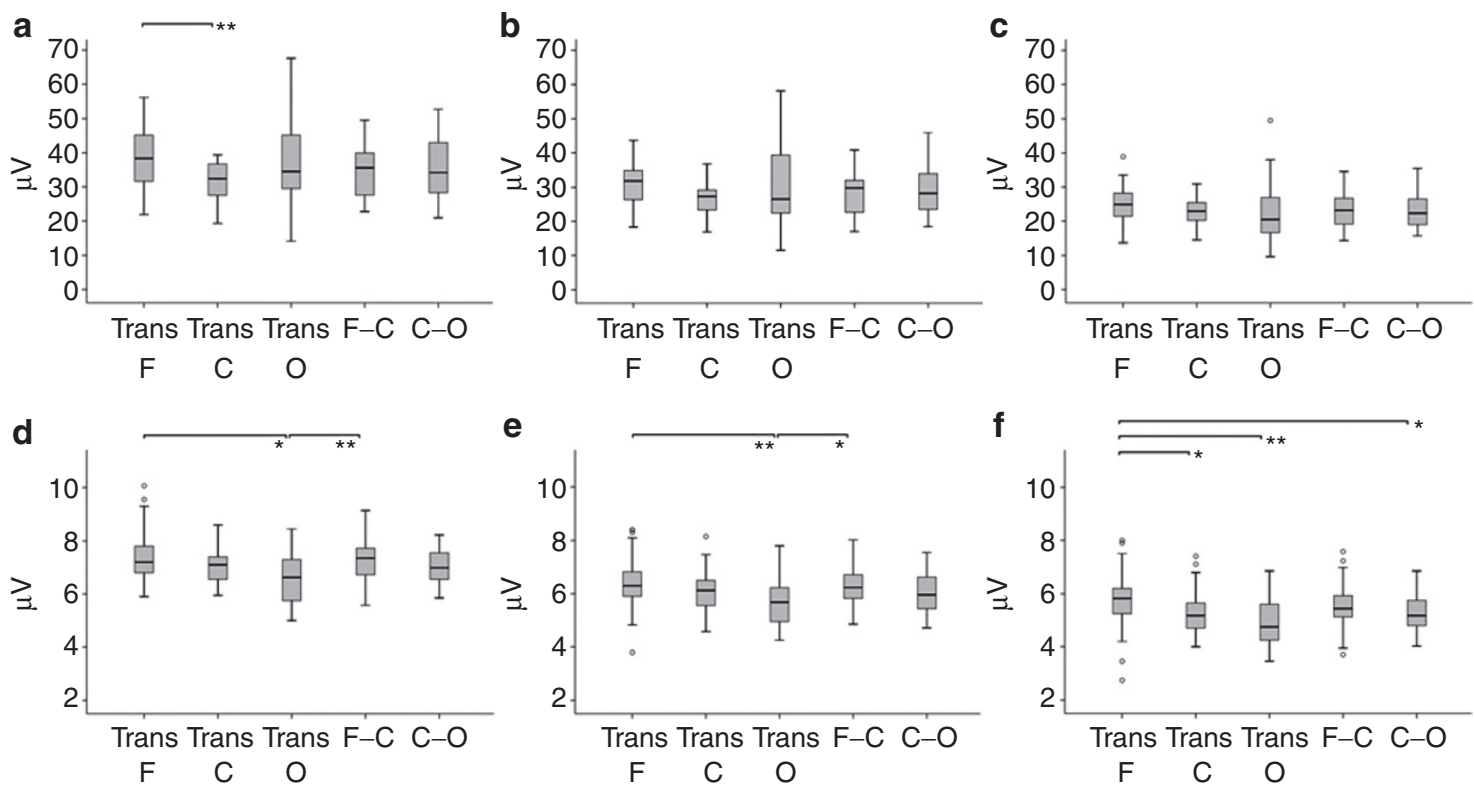

Figure 1. Box plots of each measurement item from the respective electrode locations at 30-32 wk post-conceptional age. (a) Peak of median value of upper-margin amplitude every 5 min (Med-UMA5). (b) Median of Med-UMA5. (c) Bottom of Med-UMA5. (d) Peak of median value of lower-margin amplitude every 5 min (Med-LMA5). (e) Median of Med-LMA5. (f) Bottom of Med-LMA5. The lower and upper edges of the box represent quartiles, the lines in the middle of the box represent the median, the whiskers represent 1.5 box lengths from the upper or the lower edge of the box, and the circles represent outliers, beyond 1.5 box lengths from the edge of the box. Trans means bilateral. C, central; F, frontal; O, occipital. ${ }^{*} P<0.05$; ${ }^{* *} P<0.01$ (Steel-Dwass test for multiple comparisons).

Table 2. Quantitative analyses of amplitude on each location of electrodes at term-equivalent age

\begin{tabular}{lcccccc}
\hline & Trans-F & Trans-C & Trans-O & F-C & C-O \\
\hline Peak of Med-UMA5 $(\mu \mathrm{V})$ & $30.0(21.6-40.7)$ & $29.2(19.3-41.7)$ & $24.3(17.0-35.9)$ & $29.1(23.4-39.8)$ & $29.3(21.1-40.1)$ & $<0.001$ \\
Median of Med-UMA5 $(\mu \mathrm{V})$ & $22.7(15.0-30.9)$ & $22.5(16.4-30.2)$ & $19.2(14.5-29.2)$ & $22.8(17.7-30.2)$ & $23.2(16.4-30.3)$ & $<0.001$ \\
Bottom of Med-UMA5 $(\mu \mathrm{V})$ & $18.6(13.2-24.3)$ & $18.0(14.1-24.6)$ & $15.6(10.2-24.5)$ & $18.0(14.1-24.3)$ & $18.7(14.2-25.2)$ & 0.003 \\
Peak of Med-LMA5 $(\mu \mathrm{V})$ & $8.0(6.2-10.4)$ & $8.5(7.1-15.3)$ & $7.5(5.8-9.8)$ & $8.6(7.2-13.4)$ & $8.7(7.2-13.1)$ & $<0.001$ \\
Median of Med-LMA5 $(\mu \mathrm{V})$ & $7.1(5.3-8.6)$ & $7.9(6.3-11.1)$ & $6.9(5.1-9.0)$ & $7.7(5.8-9.7)$ & $8.0(6.1-10.0)$ & $<0.001$ \\
Bottom of Med-LMA5 $(\mu \mathrm{V})$ & $5.9(4.2-7.7)$ & $7.2(4.3-9.1)$ & $5.9(4.1-8.2)$ & $6.7(5.1-9.3)$ & $7.1(4.7-8.9)$ & $<0.001$ \\
\hline
\end{tabular}

All values are shown as medians (range). Trans means bilateral.

C, central; F, frontal; Med-LMA5, median value of lower-margin amplitude every 5 min; Med-UMA5, median value of upper-margin amplitude every 5 min; O, occipital.

aKruskal-Wallis test.

that the amplitude for the C3-C4 electrodes was significantly higher than that for the P3-P4 electrodes on aEEG in 10 neonates with hypoxic ischemic encephalopathy. Their data are consistent with our results, although our study showed more detail with regard to the amplitude difference, and we used more electrode channels in a larger number of infants. One reason for these differences in amplitude seen among the electrode locations is technical, because increasing interelectrode distances significantly increase the aEEG amplitude (10). The distances of the trans-frontal or trans-occipital electrodes are shorter than those of the trans-central, fronto-central, or centro-occipital electrodes. Another reason is the ontogeny of the EEG. Different changes in the amplitude of various EEG components among the brain locations occur in preterm infants with brain maturation. Although details of these changes have rarely been reported, we previously reported the maturational changes in the amplitude of delta/theta/alpha/beta waves on conventional EEG in several electrode locations for preterm infants using amplitude spectral analysis $(11,12)$. We also reported that many components of the conventional EEG in preterm infants changed markedly with brain maturation (13). These data support this explanation. We recorded quantitative analyses of the peak, median, and bottom of the median values of UMA and LMA every 5 min because aEEG usually shows cycling that is characterized by smooth sinusoidal variations, primarily in the LMA, after 29-30 wk PCA (1). We considered that it was important to evaluate aEEG on the basis of cycling because the UMA and LMA should be affected by cycling. In our quantitative analyses, the peak and bottom values could reflect that cycling.

In this study, the locational differences in amplitude changed between preterm age and TEA. The main changes were depression of Med-UMA5 in the trans-occipital lead and depression of Med-LMA5 in the trans-frontal lead. The reason that these 

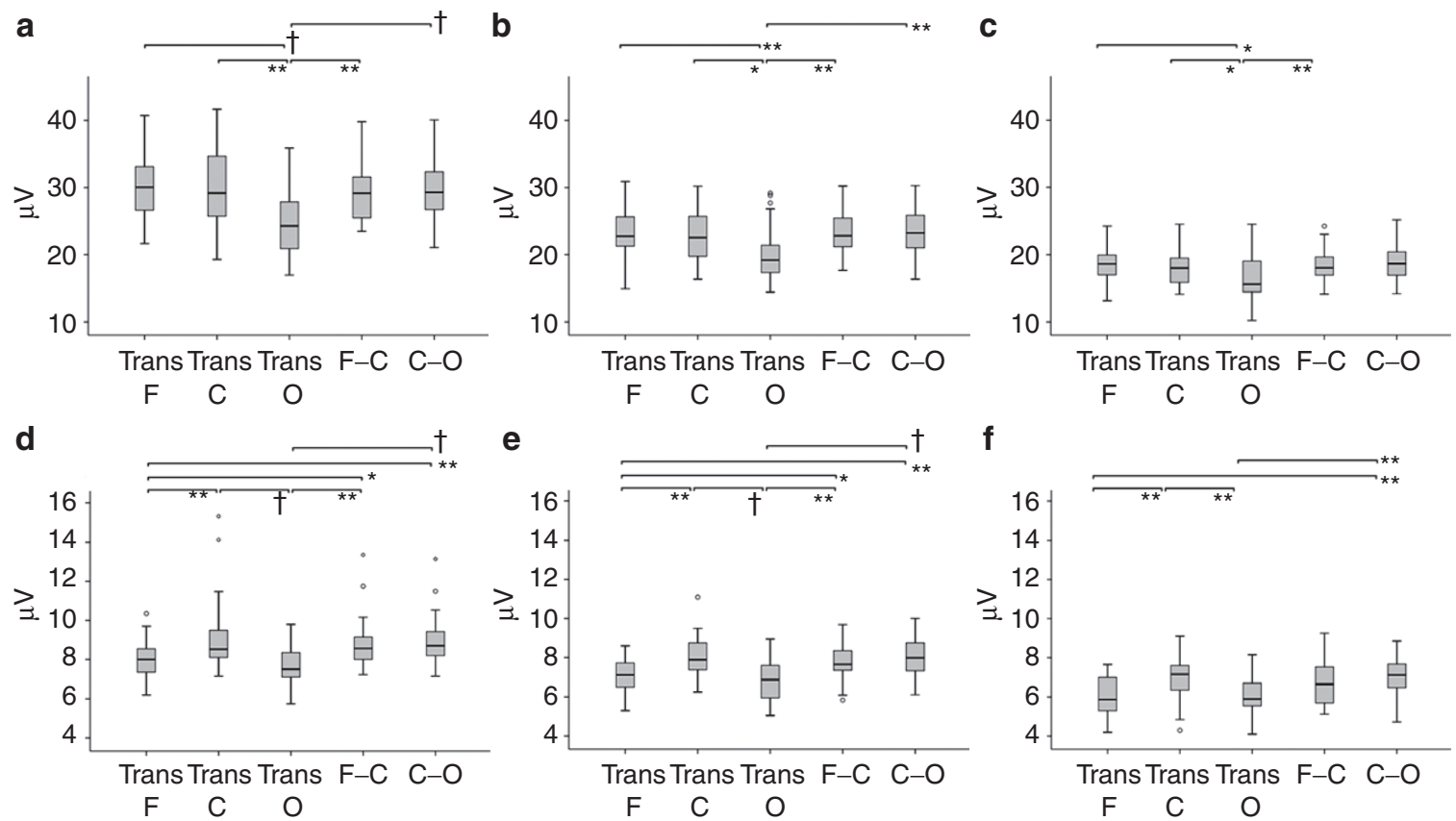

Figure 2. Box plots of each measurement item from the respective electrode locations at term-equivalent age. (a) Peak of median value of upper-margin amplitude every 5 min (Med-UMA5). (b) Median of Med-UMA5. (c) Bottom of Med-UMA5. (d) Peak of median value of lower-margin amplitude every 5 min (Med-LMA5). (e) Median of Med-LMA5. (f) Bottom of Med-LMA5. The lower and upper edges of the box represent quartiles, the lines in the middle of the box represent the median, the whiskers represent 1.5 box lengths from the upper or the lower edge of the box, and the circles represent outliers, beyond 1.5 box lengths from the edge of the box. Trans means bilateral. $\mathrm{C}$, central; $\mathrm{F}$, frontal; $\mathrm{O}$, occipital. ${ }^{*} P<0.05$; ${ }^{* *} P<0.01 ;+P<0.001$ (Steel-Dwass test for multiple comparisons).

changes were observed between preterm age and TEA is unclear, but it could be generated by the ontogeny of the EEG, as mentioned earlier. To our knowledge, no previous study has demonstrated differences in amplitude among electrode locations in preterm infants or changes in locational amplitude between preterm age and TEA. It is important to understand these differences and changes to evaluate aEEGs in preterm infants precisely.

Many studies have demonstrated the prognostic value of aEEG in term infants with hypoxic ischemic encephalopathy, and the value of LMA is one of the most important criteria in such assessments (2-4). In our study, we evaluated aEEGs only in preterm infants. However, we considered that the records at TEA would show the same results as those of term infants because the aEEG maturation of preterm infants would parallel PCA, as we reported previously (13). In our study, all LMA measurement items from the trans-frontal electrodes were significantly lower at TEA than those from the trans-central electrodes. Therefore, if trans-frontal electrodes were selected on aEEG recording in term infants with hypoxic ischemic encephalopathy, underestimation of amplitudes might occur. Appropriate electrode locations with appropriate criteria for evaluation are important in assessing aEEGs in infants, especially those with pathological conditions. This study may show that use of the trans-frontal electrodes on aEEG could be inappropriate in term infants or preterm infants at TEA according to previously reported criteria (1-4).

This study has several limitations. First, the number of channels analyzed was limited to seven, and parietal electrodes were not used because we routinely record conventional EEGs using eight electrodes (AF3, AF4, C3, C4, O1, O2, T3, and T4). Second, a problem with artifacts was observed on aEEGs in preterm infants, as reported previously (14). One record was excluded mainly because of such artifacts. However, we removed artifacts as much as possible using both quantitative and visual analyses.

In conclusion, this study demonstrated that the amplitude on aEEG was significantly different among the electrode locations in preterm infants and that such locational differences in amplitude showed changes between preterm age and TEA. It is important to understand these differences for precise evaluations. Further study is needed with more electrode channels to evaluate more precisely differences in amplitude among electrode locations.

\section{METHODS}

\section{Patients}

In total, 70 infants whose gestational age was between 27 and $32 \mathrm{wk}$ were admitted to the neonatal intensive care unit of Okazaki City Hospital between December 2008 and July 2011. Of these, 32 infants were excluded for the following reasons: insufficient EEG records in 21 (no record at TEA in 20, too many artifacts in one), congenital anomalies in three, intraventricular hemorrhage or periventricular leukomalacia in three, cardiopulmonary instability in two, respiratory instability in one, severe infection in one, and conventional EEG abnormalities as defined by our previously reported criteria (15-17) in one. Ultimately, 38 clinically stable preterm infants were included, none of whom had received sedative medications around the aEEG recording.

Cranial ultrasonography using a SONOS 5500 with an s12 transducer (Philips Medical Systems, Andover, MA) or ACUSON X-300 with a P9-4 transducer (Siemens Healthcare, Malvern, PA) was 
administered almost every day during the first week of life and at least once a week thereafter in all subjects until discharge. All infants had normal findings according to previously reported criteria (18). Brain magnetic resonance imaging was also performed at TEA in all infants using a Signa HDxt 1.5-Tesla imager (GE Healthcare, Buckinghamshire, UK) or a Sigma Horizon LX 1.5-Tesla imager (Yokokawa GE Medical, Tokyo, Japan) with conventional T1-weighted, T2-weighted, and fluid-attenuated inversion recovery imaging. All infants had normal findings according to the previously reported criteria (19).

\section{aEEG Recordings}

In our neonatal intensive care unit, conventional EEGs were routinely obtained within $3 \mathrm{~d}$ of birth, between 7 and $10 \mathrm{~d}$ of age, and then at 2-wk intervals until discharge. Conventional EEGs were recorded polygraphically at the bedside, usually for $60 \mathrm{~min}$, using a bipolar montage with at least eight surface $\mathrm{Ag} / \mathrm{AgCl}$ cup electrodes (AF3, AF4, C3, C4, O1, O2, T3, and T4) according to the international 10-20 system with a NicoletOne Monitor nICU (CareFusion, San Diego, CA). Seven-channel (AF3-AF4, C3-C4, O1-O2, AF3-C3, AF4-C4, C3-O1, and C4-O2) aEEGs were obtained simultaneously with the conventional EEG both at 30-32 wk PCA and at TEA using a standard protocol (1). The impedance was usually $<20 \mathrm{k} \Omega$. Elefix (Nihon Kohden, Tokyo, Japan) was used as electrode paste.

Informed consent for the EEG recordings was obtained from the parents of all patients. Approval for the study was obtained from the institutional review board at Okazaki City Hospital.

\section{aEEG Assessments}

For quantitative analyses, we used the following method, modified from the previously reported method of Zhang et al. (20). We outputted the UMA and the LMA data from all seven channels for the entire record as a text file from the NicoletOne Monitor, with a time resolution of $1 \mathrm{~s}$. Then the data were divided into epochs of $15 \mathrm{~s}$, and the maximum of UMA and minimum of LMA were extracted in accordance with the standard clinical aEEG trace. Then we calculated MedUMA5 and Med-LMA5. Finally, we extracted the peak, median, and bottom values of Med-UMA5 and Med-LMA5 (Figure 3a,b). UMA

a
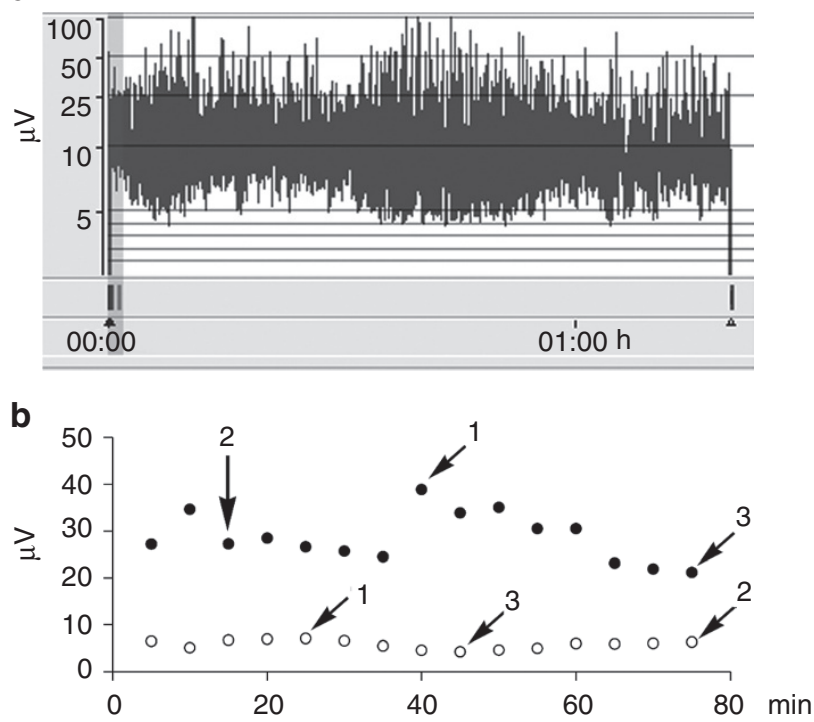

Figure 3. Quantitative analyses of amplitude-integrated electroencephalograms (aEEGs). (a) Representative aEEG on AF3-AF4 in an infant of 28 wk of gestation at $32 \mathrm{wk}$ post-conceptional age using a NicoletOne Monitor. (b) Scatter plots of median values of upper-margin amplitude (filled circles) and lower-margin amplitudes (open circles) every $5 \mathrm{~min}$ of the same record. Arrows 1, 2, and 3 show peak, median, and bottom of each value, respectively. data beyond $100 \mu \mathrm{V}$ were removed automatically as artifacts with LMA data of the same epochs, and the other data with artifacts were removed visually according to the raw EEG or aEEG waveforms.

The calculated values from the AF3-C3 and AF4-C4 channels were averaged into the values of front-central lead, and those of C3-O1 and $\mathrm{C} 4-\mathrm{O} 2$ were also averaged into the values of centro-occipital lead. Finally, we compared the aforementioned aEEG values among the trans-frontal (AF3-AF4), trans-central (C3-C4), trans-occipital (O1-O2), fronto-central (averaged values of AF3-C3 and AF4-C4), and centro-occipital (averaged values of $\mathrm{C} 3-\mathrm{O} 1$ and $\mathrm{C} 4-\mathrm{O} 2$ ) electrodes.

\section{Statistical Analysis}

Data were analyzed with the R software package (ver. 2.8.1; http:// www.R-project.org). The Kruskal-Wallis test was used to compare the difference in amplitude among the locations of lead sites. If there was a significant difference, a Steel-Dwass analysis was used for multiple comparisons. $P$ values $<0.05$ were deemed to indicate statistical significance.

\section{STATEMENT OF FINANCIAL SUPPORT}

No financial assistance was received to support this study.

Disclosure: The authors have no conflicts of interest.

\section{REFERENCES}

1. Hellström-Westas L, Rosén I. Continuous brain-function monitoring: state of the art in clinical practice. Semin Fetal Neonatal Med 2006;11: 503-11.

2. Shalak LF, Laptook AR, Velaphi SC, Perlman JM. Amplitude-integrated electroencephalography coupled with an early neurologic examination enhances prediction of term infants at risk for persistent encephalopathy. Pediatrics 2003;111:351-7.

3. ter Horst HJ, Sommer C, Bergman KA, Fock JM, van Weerden TW, Bos AF. Prognostic significance of amplitude-integrated EEG during the first 72 hours after birth in severely asphyxiated neonates. Pediatr Res 2004;55:1026-33.

4. van Rooij LG, Toet MC, Osredkar D, van Huffelen AC, Groenendaal F, de Vries LS. Recovery of amplitude integrated electroencephalographic background patterns within 24 hours of perinatal asphyxia. Arch Dis Child Fetal Neonatal Ed 2005;90:F245-51.

5. Hellström-Westas L, Klette H, Thorngren-Jerneck K, Rosén I. Early prediction of outcome with aEEG in preterm infants with large intraventricular hemorrhages. Neuropediatrics 2001;32:319-24.

6. Olischar M, Klebermass K, Waldhoer T, Pollak A, Weninger M. Background patterns and sleep-wake cycles on amplitude-integrated electroencephalography in preterms younger than 30 weeks gestational age with peri-/intraventricular haemorrhage. Acta Paediatr 2007;96:1743-50.

7. Bowen JR, Paradisis M, Shah D. Decreased aEEG continuity and baseline variability in the first 48 hours of life associated with poor shortterm outcome in neonates born before 29 weeks gestation. Pediatr Res 2010;67:538-44.

8. Shellhaas RA, Soaita AI, Clancy RR. Sensitivity of amplitude-integrated electroencephalography for neonatal seizure detection. Pediatrics 2007;120:770-7.

9. Bourez-Swart MD, van Rooij L, Rizzo C, et al. Detection of subclinical electroencephalographic seizure patterns with multichannel amplitude-integrated EEG in full-term neonates. Clin Neurophysiol 2009;120:1916-22.

10. Quigg M, Leiner D. Engineering aspects of the quantified amplitudeintegrated electroencephalogram in neonatal cerebral monitoring. J Clin Neurophysiol 2009;26:145-9.

11. Okumura A, Kubota T, Toyota N, et al. Amplitude spectral analysis of maturational changes of delta waves in preterm infants. Brain Dev 2003;25:406-10.

12. Okumura A, Kubota T, Tsuji T, Kato T, Hayakawa F, Watanabe K. Amplitude spectral analysis of theta/alpha/beta waves in preterm infants. Pediatr Neurol 2006;34:30-4. 


\section{Articles $\mid$ Kato et al.}

13. Kato T, Okumura A, Hayakawa F, Tsuji T, Natsume J, Watanabe K. Evaluation of brain maturation in pre-term infants using conventional and amplitudeintegrated electroencephalograms. Clin Neurophysiol 2011;122:1967-72.

14. Suk D, Krauss AN, Engel M, Perlman JM. Amplitude-integrated electroencephalography in the NICU: frequent artifacts in premature infants may limit its utility as a monitoring device. Pediatrics 2009;123:e328-32.

15. Watanabe K, Hayakawa F, Okumura A. Neonatal EEG: a powerful tool in the assessment of brain damage in preterm infants. Brain Dev 1999;21:361-72.

16. Maruyama K, Okumura A, Hayakawa F, Kato T, Kuno K, Watanabe K. Prognostic value of EEG depression in preterm infants for later development of cerebral palsy. Neuropediatrics 2002;33:133-7.
17. Kidokoro H, Okumura A, Hayakawa F, et al. Chronologic changes in neonatal EEG findings in periventricular leukomalacia. Pediatrics 2009;124:e468-75.

18. O'Shea TM, Kuban KC, Allred EN, et al. Neonatal cranial ultrasound lesions and developmental delays at 2 years of age among extremely low gestational age children. Pediatrics 2008;122:e662-9.

19. Counsell SJ, Rutherford MA, Cowan FM, Edwards AD. Magnetic resonance imaging of preterm brain injury. Arch Dis Child Fetal Neonatal Ed 2003;88:F269-74.

20. Zhang D, Liu Y, Hou X, et al. Reference values for amplitude-integrated EEGs in infants from preterm to 3.5 months of age. Pediatrics 2011;127:e1280-7. 